\title{
ATLAS Trigger Status and Results From Commissioning Operations
}

\author{
Michela Biglietti ${ }^{1}$ on behalf of the ATLAS Collaboration \\ University of Napoli and INFN \\ Dipartmento di Scienze Fisiche, via Cintia, I-80126, Napoli, Italy. \\ E-mail: michela.bigliettiecern.ch
}

\begin{abstract}
The ATLAS trigger system is designed to select rare physics processes from an extremely high rate of proton-proton collisions. The short LHC bunch crossing period of $25 \mathrm{~ns}$ and the large background of soft-scattering events overlapped in each bunch crossing pose serious challenges, both on hardware and software, that the ATLAS trigger must overcome in order to efficiently select interesting events. The ATLAS trigger consists of a hardware based Level-1, and a twolevel software based High-Level Trigger (HLT). Data bandwidth and processing times in the higher level triggers are reduced by region of interest guidance in the HLT reconstruction steps. High flexibility is critical in order to adapt to the changing luminosity, backgrounds and physics goals. It is achieved by the use of inclusive trigger menus and modular software design. Selection algorithms have been developed which provide the required elasticity to detect different physics signatures and to control the trigger rates. In this paper an overview of the ATLAS trigger design, status and expected performance, as well as the results from the ongoing commissioning with cosmic rays and first LHC beams, is presented.
\end{abstract}

XII Advanced Computing and Analysis Techniques in Physics Research

Erice, Italy

3-7 November, 2008

${ }^{1}$ Speaker 


\section{Introduction}

The ATLAS experiment $[1,2]$ is one of the four experiments at the Large Hadron Collider (LHC), that is scheduled to start to operate in the year 2009. At present, the installation of the different components of the ATLAS detector is completed in the underground cavern and the commissioning process has already started. At the design luminosity of $10^{34} \mathrm{~cm}^{-2} \mathrm{~s}^{-1}$ the detector will be exposed every $25 \mathrm{~ns}$ to, on average, 25 proton-proton interactions. These conditions make the environment for the trigger system extremely demanding. It has to reduce the input event rate of $40 \mathrm{MHz}$ to the limit of the storage rate of $200 \mathrm{~Hz}$ or less. The ATLAS trigger is composed of three levels: Level 1 (L1) [3] which is hardware-based, the Level 2 (L2) and Event Filter (EF) (collectively referred to as the High Level Trigger or HLT [4]) based on software algorithms analyzing the data on large computing farms. This note focuses on the trigger strategy that can be deployed during the initial low luminosity running and describes the commissioning operations of the Trigger for initial running with cosmic rays and first LHC beams.

\section{The ATLAS Detector and Trigger Strategy}

In order to enrich the new-physics content of the recorded data (and remain within the limits of technology to data transfer) a data selection is made through the use of a highly-selective threelevel trigger. The $\mathrm{L} 1$ reduces the $40 \mathrm{MHz}$ input rate (bunch-crossing rate) to about $75 \mathrm{kHz}$ (upgradable to $100 \mathrm{kHz}$ ). It uses coarse granularity data from the muon and calorimeter detector systems. L1 must reach a decision within $2.2 \mu \mathrm{s}$. The L1 selection is mainly based on the identification of high transverse momentum objects in the detector.

The allowed L1 configurations (also called L1 items) are programmed in the Central Trigger Processor (CTP) [5]; each of L1 items is a logical combination of a given multiplicities of one or more of the configured L1 thresholds.

The L2 reconstruction is seeded by the L1 Regions of Interest (RoIs) and has access to the full detector granularity. The size of the RoI is determined by the L2 algorithms depending on the type of triggered object. RoI mechanism is used to minimize the amount of data needed to calculate the trigger decisions thus reducing the overall network data traffic considerably. The trigger requests only a few percent of the detector data, leading to large savings in the necessary network bandwidth. Within each RoI, the L2 reconstructs physics objects using fast algorithms that use detector information not available at L1, like data from Muon Spectrometer precision chambers and the Inner Detector. The information from individual sub-systems can then be matched to provide additional rejection and higher purity. The L2 farm will consist of around 500 quad-core CPUs; the average processing time at L2 is $40 \mathrm{~ms}$ and the expected output rate is around $2 \mathrm{kHz}$.

The EF is subsequently seeded by L2. The EF has, on average, $4 \mathrm{~s}$ to process each event. This allows the use of the more sophisticated offline reconstruction algorithms, as well as offline-like calibration and alignment corrections. The Event Filter algorithms run on a farm of 1800 dual quad-core CPUs processors. The output rate of the EF is limited to $200 \mathrm{~Hz}$, assuming an event 
size of $1.5 \mathrm{Mb}$. The execution of the HLT algorithms is organized by the HLT Steering [4] based on the static configuration information and on the dynamic event data. The configuration contains a list of the active signatures (trigger menu) and their thresholds, pass-through fractions and prescale factors. The HLT algorithm sequences are divided into reconstruction steps followed by verification steps. The chain of algorithms can be stopped at any of the verification steps if it is found to be non-viable (early rejection), thus freeing resources for the next signature.

The HLT algorithms are logically divided into groups of related signatures. Currently these are known loosely as: e/ $\gamma, \mu, \tau$, jets, b-tag, B physics, missing ET, cosmics and minimum bias. Algorithms from one or more groups, together with configuration information such as threshold values and prescale factors are used to build the trigger signatures that form the trigger menu.

\section{Expected Performance in Early Running}

During the LHC startup phase, where low luminosity conditions $\left(10^{31} \mathrm{~cm}^{-2} \mathrm{~s}^{-1}\right.$ or less $)$ and low number of bunches in the machine are expected to prevail, the focus of the trigger selection strategy will be to commission the trigger and the detector and to ensure that established Standard Model processes are observed.

In the very early operations, many triggers, especially those at higher thresholds, will operate in pass-through mode, which entails executing the trigger algorithms but accepting the event independent of the decision. This allows the trigger selections and algorithms to be validated to ensure that they are robust against the varying beam and detector conditions that are hard to predict before data taking. As the luminosity increases, the use of higher thresholds, isolation criteria and tighter selections at HLT become necessary to reduce the background rates while achieving selection of interesting physics with high efficiency.

An initial trigger menu has been designed for a luminosity of $10^{31} \mathrm{~cm}^{-2} \mathrm{~s}^{-1}$ using simulated minimum-bias events. The following notation is used to label the different trigger items: EM (electromagnetic), J (jets), MU (muons), and tau (tau leptons). Table 1 shows a representative set of L1 signatures, their prescale factors and estimated rates during the start-up for an assumed luminosity of $10^{31} \mathrm{~cm}^{-2} \mathrm{~s}^{-1}$. At low luminosities, a single non-isolated EM trigger with threshold of $7 \mathrm{GeV}$ can be used without the application of prescale factors at the first trigger level with a rate of about $5 \mathrm{kHz}$. The total $\mathrm{L} 1$ output rate out for the EM objects is about $10 \mathrm{kHz}$. For the jet items, a single trigger with a threshold of $120 \mathrm{GeV}$ can be deployed without prescale factors and has a L1 output rate of about $9 \mathrm{~Hz}$. Single muon and di-muon signatures could all be deployed at $10^{31} \mathrm{~cm}^{-2} \mathrm{~s}^{-1}$ without prescale factors with further selection at HLT to control the output rates. Tau triggers at low luminosity are chosen to collect large statistics of $W$ and $Z$ boson decays. The reliance on missing energy end total energy trigger will be small, especially during the early running period, as it is sensitive to various detector effects that will require time to understand. 
Table 1: A representative list of $L 1$ trigger items and estimated rates at $10^{31} \mathrm{~cm}^{-2} \mathrm{~s}^{-1}$

\begin{tabular}{|l|c|c|c|c|c|c|c|c|c|}
\hline Trigger Item & EM3 & EM7 & 2EM3 & MU4 & MU20 & J10 & J120 & tau6 & tau40 \\
\hline Prescale & 60 & 1 & 1 & 1 & 1 & 42000 & 1 & 750 & 1 \\
\hline Rate $(\mathrm{Hz})$ & 674 & 4900 & 6500 & 1730 & 20 & 4 & 9 & 19 & 83 \\
\hline
\end{tabular}

Most of the trigger items in the L2 and EF are either executed in pass-through mode or with loose selections. Figure 1 shows a summary of the EF output rates for each trigger group and the cumulative rates, which provide a running total of the rates. The sum of the rates for all the trigger groups is more than the cumulative rates due to overlaps between the groups. The total cumulative rates for this trigger menu, accounting for overlaps between the trigger groups, is $12 \mathrm{kHz}$ at L1, $620 \mathrm{~Hz}$ at L2 and $197 \mathrm{~Hz}$ at EF. Since there are large uncertainties related to the simulation, the estimated rate out of L1 and L2 is kept well below their respective targets of 40 $\mathrm{kHz}$ and $1 \mathrm{kHz}$ available during the LHC startup phase. The EF selections have been tuned to yield the targeted EF output rate compatible with the design output rate of $200 \mathrm{~Hz}$; this preliminary trigger list will need to be optimized based on early experience with real data.

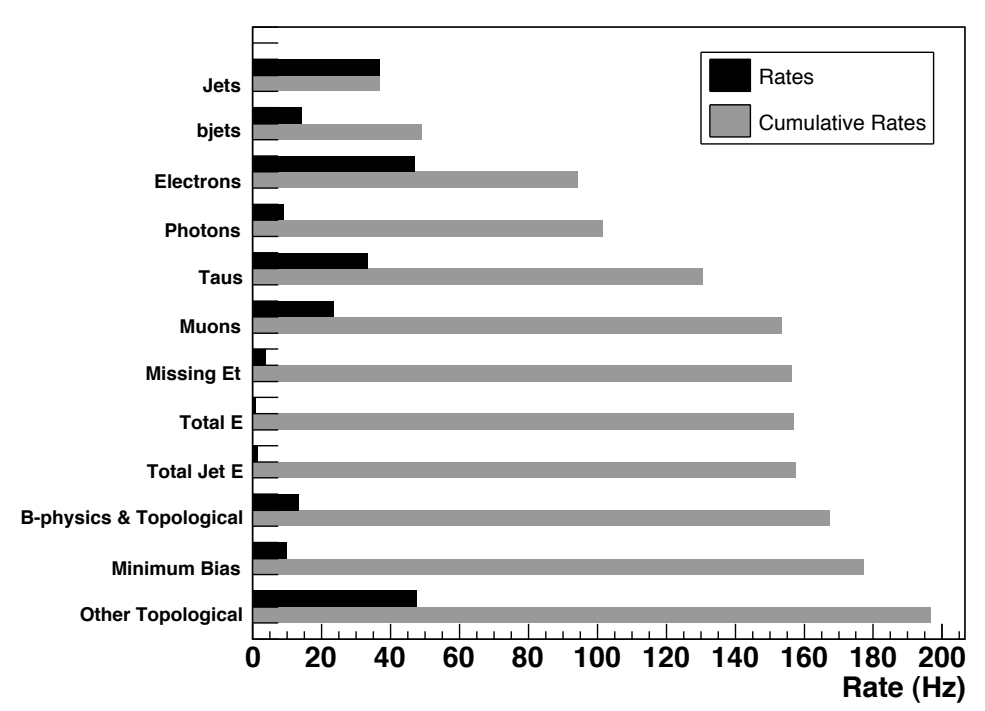

Figure 1: HLT unique (black) and cumulative (gray) estimated rates at $10^{31} \mathrm{~cm}^{-2} \mathrm{~s}^{-1}$ for different trigger groups.

\section{Commissioning the ATLAS Trigger with Cosmic Rays and First Beam}

During the last two years (2007 and 2008), the focus of ATLAS commissioning effort has evolved from single detector operation to combined running and integration. Several combined tests (called Milestone Runs) with cosmic rays have been scheduled to integrate detector, trigger and data acquisition into one global setup for each group of sub-systems (calorimeters, muon 
detectors, inner detector and magnets). Milestone Runs have been very helpful in the commissioning of the subsystems, in the integration of controls and of the trigger and data acquisition. Moreover, ATLAS was operational when the LHC beams circulated for the first time in September 2008. To cope with different commissioning phases, special trigger menus have been prepared with selection criteria suited to cosmic-ray events, beam-halo and low intensity collisions, and where the trigger is mainly based on L1 items with loose requirements on single tracks and energy clusters. Since the cosmic rays do not come from the interaction point but top of the ATLAS cavern, to increase the acceptance, the trigger condition for the L1 muon trigger was set with the largest possible coincidence widows. This means that the trigger roads for the lower thresholds follow the cabling layout. L1 calorimeter trigger was not designed to be able to trigger on cosmic muons since the noise in each trigger tower is comparable to the signal given by a minimum-ionizing particle. Nonetheless, cosmics with energy deposits greater than few $\mathrm{GeV}$ could be triggered. Figure 2 shows the integrated cosmic data recorded by ATLAS with different triggers, versus the time for one of the most active period, i.e. after Sep 13, 2008. For most of the runs the full ATLAS detector (except for CSC) was included in the data acquisition. In some runs the Liquid-Argon Calorimeter was taken out, allowing trigger to accept rates of approximately $500 \mathrm{~Hz}$ (corresponding to the high rises in the curves). During the cosmics run the HLT infrastructure and algorithms were operational and used in a transparent trigger mode, where events are tagged with HLT results, but not discarded accordingly, as it would happen in standard operation. In addition to this, there is an active HLT selection performed based on tracking algorithms in order to enrich selected streams with tracks useful for timing and alignment studies.

On September $10^{\text {th }} 2008$, and later on for few days, the first beams of protons in single bunches have been circulated successfully in the LHC. For safety considerations, ATLAS has taken the data with the Pixel detector was switched off, and some other subsystems were operated with reduced voltage. The solenoid magnet was off, but the toroid systems were operational.

During this phase, in addition to L1 Calo and Muon, two tools have been set-up for early beams: a beam pick-up from the accelerator (BPTX), and a minimum-bias trigger (MBTS) derived from hodoscopes of plastic scintillators installed on the front face of the end-cap calorimeters. The events recorded by ATLAS with the first beams circulating in LHC can be classified as high-multiplicity events, when the beams were dumped on collimators at $140 \mathrm{~m}$ from ATLAS, and halo events, when the collimators were open and fewer particles from beam interactions crossed the detector. These events provide useful checks as, for example, the trigger timing alignment with respect to BPTX. Figure 3 shows the relative time distribution for the different TGC triggers, with the time-of-flight effect particularly visible. The TGC wheels are located at $\mathrm{z}$ $= \pm 14 \mathrm{~m}$, so the two trigger planes generate signals with time difference of about $100 \mathrm{~ns}$, corresponding to 4 bunch crossing units. 


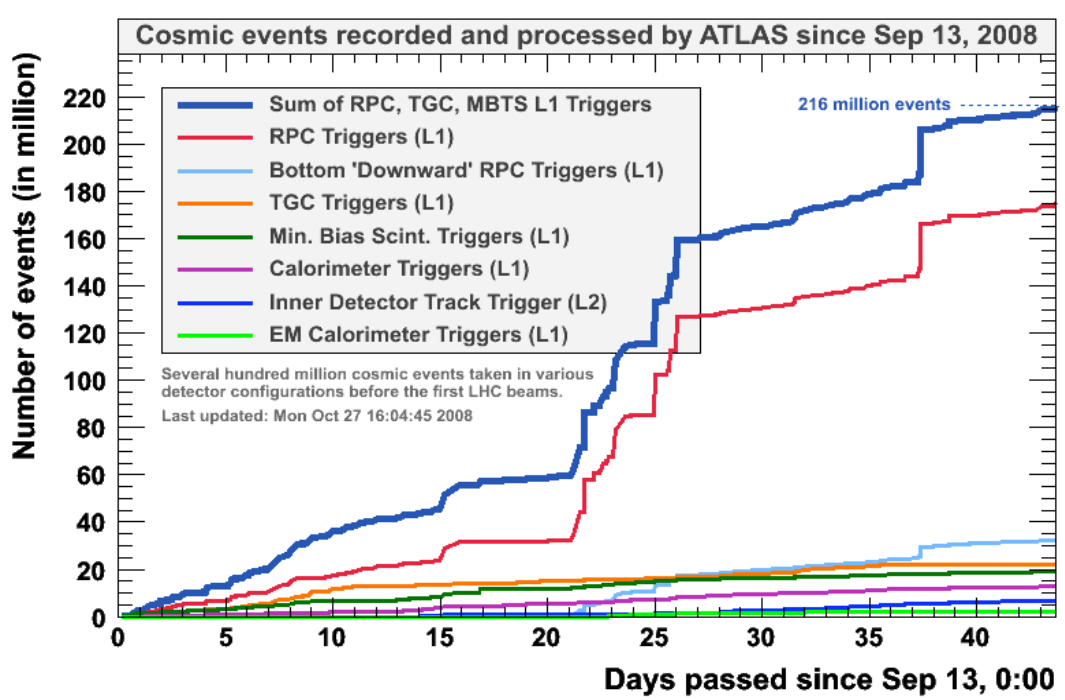

Figure 2: Integrated cosmic data rate for ATLAS versus time after Sep 13, 2008.

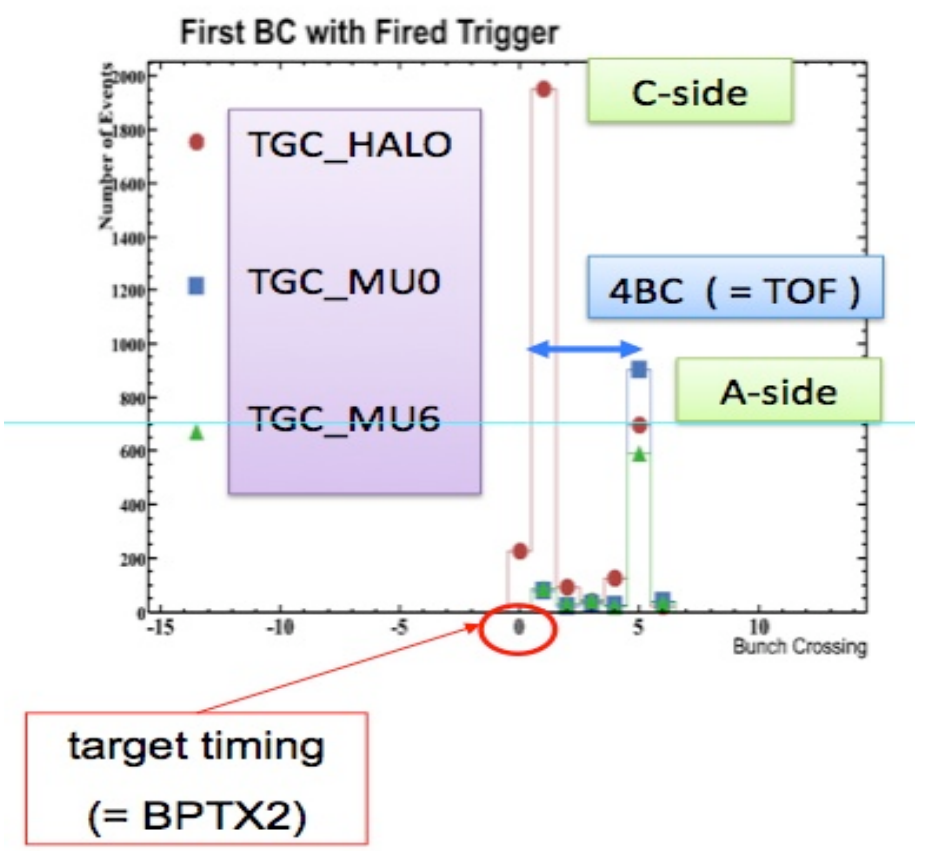

Figure 3 :TGC trigger timing distribution with single-beam with respect to bunch-crossing units (25 ns). 


\section{Conclusions}

The three levels of the ATLAS trigger have been designed to handle the LHC rates and occupancies at high luminosity. The ATLAS trigger and its performance have been studied in detail both analyzing the performance with simulated data in the initial LHC scenario and operating the system with cosmic runs. The LHC startup in September 2008 found the ATLAS detector ready; LHC data have been recorded and analyzed providing useful information. ATLAS is looking forward to collect data from LHC collisions.

\section{References}

[1] The ATLAS Collaboration, ATLAS Technical Proposal, CERN/LHCC/94-43, December 1994

[2] The ATLAS Collaboration, The ATLAS Experiment at the CERN Large Hadron Collider, JINST (2008) 3 S08003;

[3] ATLAS Collaboration, Level 1 Trigger Technical Design Report, CERN/LHCC/98-14 (1998).

[4] ATLAS Collaboration, High-Level Trigger, Data Acquisition and Controls Technical Design Report, CERN/LHCC/03-022 (2003).

[5] R. Spiwoks et al, The ATLAS level-1 central trigger processor core module (CTP_CORE), IEEE Trans. Nucl. Sci. 52 (2005) 3211-3215. 